\title{
ON CONTINUOUS REGULAR RINGS AND SEMISIMPLE SELF INJECTIVE RINGS
}

\author{
YUZO UTUMI
}

1. Introduction. Brainerd and Lambek (2, Corollary 4) have proved recently that any complete Boolean ring is self-injective. It is easy to see that every complete Boolean ring is a continuous regular ring, that is, a regular ring of which the lattice of principal left ideals is continuous. This suggests that in a continuous regular ring it might be possible to prove the injectivity. However, a simple example (Example 3 ) shows that the conjecture is not true in general. Our main theorem is the following. Every continuous regular ring with no ideals of index 1 is (both left and right) self-injective (Theorem 3).

It is known to Wolfson (13, Theorem 5.1) and Zelinsky (15) that the ring $S$ of all linear transformations of a vector space of dimension $\geqslant 2$ over a division ring is generated by idempotents and also by non-singular elements. We shall in the present paper prove this under the assumption that the ring is a semisimple one-sided self-injective ring with no ideals of index 1 (Theorem 2). Since the above $S$ satisfies this assumption (10, (5.1)), our theorem may be regarded as a generalization of the result of Wolfson and Zelinsky.

The author wishes to express his appreciation to J. Lambek for his interest and encouragement.

2. Preliminaries. Throughout this paper the word ideal without modifier will always mean two-sided ideal.

A ring is said to be a semisimple I-ring if every non-zero left ideal contains a non-zero idempotent.

For any left ideal $A$ of a ring $S$ the least upper bound of all integers $r$ such that $A$ contains a direct sum of $r$ mutually isomorphic non-zero left ideals of $S$ will be denoted by $\mathrm{m}(A)$.

We say that a ring $S$ is of index $\mathrm{m}$, if $S$ contains nilpotent elements of index $\mathrm{m}$ (that is, $a^{\mathrm{m}-1} \neq 0, a^{\mathrm{m}}=0$ ) but no elements of higher index. We shall denote the index of $S$ by i $(S)$.

(S1) For any left ideal $A$ of a semisimple $I$-ring $S$ we have $\mathrm{m}(A)=$ $\mathrm{m}(A / \mathrm{N}(A))=\mathrm{i}(A / \mathrm{N}(A))$ where $\mathrm{N}(A)$ denotes the radical of $A$ (11, Theorem $3)$.

(S2) Every idempotent of a ring $S$ is central if $\mathrm{i}(S)=1$.

(S3) An idempotent $e$ of a ring $S$ with no nilpotent ideals is central if and only if $e S \subset S e$.

Received August 10, 1959. 
A ring is called regular if for any $x$ there is an element $y$ with $x y x=x$. The lattice of principal left (right) ideals of a regular ring $S$ will be denoted by $\mathscr{L}(S)(\mathscr{R}(S))$.

(R1) For any two idempotents $e, f$ of a regular ring $S$ if $e S f \neq 0$ then 0 $\neq S e^{\prime} \subset S e, S f^{\prime} \subset S f$ and $S e^{\prime} \simeq S f^{\prime}$ for some $e^{\prime}, f^{\prime}$.

In fact, let $0 \neq x \in e S f$. The right multiplication by $x$ gives a non-zero homomorphism $\theta: S e \rightarrow S f$. Then $\operatorname{ker} \theta(=$ the kernel of $\theta)$ is a principal left ideal. Let $S e=\operatorname{ker} \theta \oplus S e^{\prime}$ and $\theta(S e)=S f^{\prime}$. It is then evident that $S e^{\prime} \simeq S f^{\prime}$.

(R2) Let $S$ be a regular ring and suppose that $\mathscr{L}(S)$ is complete. Then $S$ has a unit.

In fact, $S=\cup_{x \in S} S x$ is generated by an idempotent $e: S e=S$. By (S3) $e$ is central and so $e=1$.

A ring $S$ is said to be a Boolean ring if $S$ satisfies the identity $x^{2}=x$. It is easily verified that the identity $x^{2}=x$ implies the identities $x y=y x$ and $x+x=0$. Every Boolean algebra may be regarded as a Boolean ring with $\mathrm{u}$ lit, and vice versa (1, p. 154).

(R3) The set of all central idempotents in a ring $S$ with unit forms a Boolean algebra (see (6, p. 49)).

For any given module $A$ and a sub-module $B$ we say that $A$ is an essential extension of $B$ if every non-zero submodule of $A$ has a non-zero intersection with $B$. Notation: $B C^{\prime} A$ (see 4 ).

A module $Q$ will be referred to as an injective module if $Q$ is a direct summand of every extension module.

If an injective module $Q$ is an essential extension of a module $A$ we say that $Q$ is a minimal injective extension of $A$. Notation: $Q=\hat{A}$.

(Q1) If $A \simeq B$, the isomorphism is extended to that of $\hat{A}$ and $\hat{B}$.

(Q2) If $A=A_{1} \oplus \ldots \oplus A_{n}$, then $\hat{A}=\hat{A}_{1} \oplus \ldots \oplus \hat{A}_{n}$.

A ring $S$ is said to be left (right) self-injective if $S$ has a unit and the left (right) $S$-module $S$ is injective. A ring which is both left and right self-injective is called a self-injective ring.

For any semisimple $I$-ring $S$ we can construct the maximal left quotient ring $\bar{S}$ of $S$ (see (7), (10), (14), and also (5)).

(Q3) $\bar{S}$ is a left self-injective regular ring.

(Q4) $\bar{S}$ is an extension ring of $S$. The left $S$-module $\bar{S}$ is an essential extension of the left $S$-module $S$.

(Q5) If $S$ has unit 1 , then 1 is also unit of $\bar{S}$.

A lattice $L$ is called upper continuous if $L$ is complete and satisfies the following

Condition (C).

$$
\left(\cup a_{\alpha}\right) \cap b=\cup\left(a_{\alpha} \cap b\right)
$$

for every chain $\left\{a_{\alpha}\right\}$ and every element $b$.

The following two conditions for continuity also may frequently be seen in the literature: 
Condition $\left(\mathrm{C}^{\prime}\right)$. (1) holds for every well-ordered ascending chain $\left\{a_{\alpha}\right\}$ and every $b\left(\mathbf{9}, \mathrm{III}_{2}\right.$, p. 3$)$.

Condition $\left(\mathrm{C}^{\prime \prime}\right)$. If a subset $\left\{a_{\alpha}\right\}$ of $L$ is directed, that is, for any $a_{\alpha}, a_{\beta}$ there is $a_{\gamma}$ with $a_{\alpha}, a_{\beta} \leqslant a_{\gamma}$, then (1) holds for every $b$ (8, Definition 1.14, p. 10).

By virtue of $\left(8, I I\right.$, p. 237) Conditions $\left(C^{\prime}\right)$ and $\left(C^{\prime \prime}\right)$ are equivalent. Evidently Condition (C) implies Condition $\left(C^{\prime}\right)$, and also Condition $\left(C^{\prime \prime}\right)$ implies Condition $(\mathrm{C})$. Therefore, these three conditions are all equivalent for every complete lattice $L$.

(C1) A complete complemented modular lattice $L$ is upper continuous if and only if $L$ satisfies the following

Condition (M). Let $T$ be a subset of $L$. If $\left(\cup_{a \in F} a\right) \cap b=0$ for every finite subset $F$ of $T$, then $\left(\cup_{a \in T} a\right) \cap b=0(8,(\alpha)$, p. 11).

In fact, by Hilfssatz 1.7 and Anmerkung 1.11 of (8, p. 11) Condition (M) is equivalent to Condition $\left(\mathrm{C}^{\prime \prime}\right)$ for any complete relatively complemented lattice $L$.

Definition. A regular ring $S$ is said to be left (right) continuous if $\mathscr{L}(S)$ $(\mathscr{R}(S))$ is upper continuous.

A continuous regular ring (8, Definition 1.1, p. 156) is a ring which is both left and right continuous. By (R2) every left (right) continuous regular ring has a unit.

We have proved in the proof of Corollary 1 of (12) the following

THEOREM 1. Every semisimple left self-injective ring is a left continuous regular ring.

COROLlary. Every semisimple self-injective ring is a continuous regular ring.

3. Generators of self-injective rings. We shall denote the left (right) annihilator of a subset $T$ of a ring by $l(T)(\mathrm{r}(T))$.

LeMmA 1. Let $S$ be a regular ring and suppose that $\mathscr{L}(S)$ is complete, then every left annihilator ideal $A$ is principal.

Proof. By (R2) $S$ has a unit 1 . Denote by $S f$ the meet of $S(1-e)$ for all idempotents $e \in \mathrm{r}(A)$. For any $x \in \mathrm{r}(A)$ there is an idempotent $e^{\prime}$ such that $x S=e^{\prime} S$. Then, $e^{\prime} \in \mathrm{r}(A)$ and $S f \subset S\left(1-e^{\prime}\right)$, whence $S f x \subset S\left(1-e^{\prime}\right) e^{\prime} S=0$ and $S f \subset l(\operatorname{r}(A))=A$. On the other hand, if $a \in A$, $a e=0$ for all idempotents $e \in \mathrm{r}(A)$, and so $a \in S(1-e)$, hence $a \in S a \subset S f$ : this implies that $A \subset S f$. Therefore, $A=S f$ is principal, as desired.

Leмma 2. Let $S$ be a left continuous regular ring. Then, for any left ideal $A$ there is a principal left ideal Se such that (1) $A \subset^{\prime}$ Se and (2) Se $\subset$ Sf whenever $A \subset S f$. In case $S$ is a semisimple left self-injective ring, Se is a minimal injective extension of $A$. 
Proof. We set $S e=\cup_{x \in A} S x$. Of course, $A \subset S e$. If $A \subset S f$, then $S x \subset S f$ for every $x \in A$, and $S e \subset S f$. Suppose that $A \cap S g=0$. For any finite subset $F$ of $A$ we have $\left(\cup_{x \in F} S x\right) \cap S g=\left(\sum_{x \in F} S x\right) \cap S g \subset A \cap S g=0$. Hence $S e \cap S g=0$ by (C1). This implies that $A C^{\prime} S e$.

Next, suppose that $S$ is a semisimple left self-injective ring. Then, by Theorem $1, S$ is a left continuous regular ring, and hence $A C^{\prime} S e$ for some $e \in S$. Since $S e$ is a direct summand of $S, S e$ is injective (as a left $S$-module) (3, p. 8). Therefore, $S e$ is the minimal injective extension of $A$, completing the proof.

Lemma 3. Let $S$ be a semisimple left self-injective ring. Suppose that (i) $\mathrm{m}(S x)=1$, (ii) $S e$ does not contain any left ideals isomorphic to $S x$, and (iii) $e=e^{2}$. Then $S(1-e)$ contains a non-zero central idempotent.

Proof. By Zorn's lemma there exists a maximal isomorphism $\theta$ of which the domain $D$ and the image $E$ are contained in $S x$ and $S e$ respectively. By Lemma $2 D$ and $E$ have minimal injective extensions $\hat{D}$ and $\hat{E}$ such that $\hat{D} \subset S x$, $\hat{E} \subset S e$ and $\hat{D}, \hat{E} \in \mathscr{L}(S)$. By (QI) $\theta$ is extended to an isomorphism of $\hat{D}$ and $E$. In view of the maximality of $\theta$ this implies that $\hat{D}=D, \hat{E}=E$, and hence also that $D, E \in \mathscr{L}(S)$. Let $E=S e^{\prime}, S e=S e^{\prime} \oplus S e^{\prime \prime}$ and $S x=D \oplus S f, e^{\prime}, e^{\prime \prime}$ and $f$ being idempotents. Now, we shall show that $f S e=0$. In fact, if $f S e^{\prime} \neq 0$, then by (Rl) there exist $S f_{1}$ and $S e_{1}{ }^{\prime}$ such that $0 \neq S f_{1} \subset S f, S e_{1}{ }^{\prime} \subset S e^{\prime}$ and $S f_{1} \simeq S e_{1}{ }^{\prime}$. It follows from this that $S x$ contains two mutually isomorphic left ideals $\theta^{-1}\left(S e_{1}{ }^{\prime}\right)$ and $S f_{1}$. Since $\left(\theta^{-1}\left(S e^{\prime}\right)\right) \cap S f \subset D \cap S f=0$, this contradicts the assumption (i). Hence $f S e^{\prime}=0$. On the other hand, if $f S e^{\prime \prime} \neq 0$, then by (R1) $0 \neq S f_{2} \subset S f, S e_{2}{ }^{\prime \prime} \subset S e^{\prime \prime}$ and $S f_{2} \simeq S e_{2}{ }^{\prime \prime}$ for some $f_{2}, e_{2}{ }^{\prime \prime} \in S$. This shows that $\theta$ may be extended to an isomorphism of $D \oplus S f_{2}$ onto $E \oplus S e_{2}{ }^{\prime \prime}$, and we obtain a contradiction. Hence $f S e^{\prime \prime}=0$. Therefore, we have $f S e=f\left(S e^{\prime}+S e^{\prime \prime}\right)=0$. Now, it is evident from the assumption (ii) that, $S f \neq 0$. Thus, $0 \neq f \in l(S e)$ and $0 \neq l(S e)$. By Theorem $1 \mathscr{L}(S)$ is complete, and hence $l(S e) \in \mathscr{L}(S)$ by Lemma 1 . Since $l(S e)$ is an ideal, $l(S e)$ is generated by a central idempotent $c$ by virtue of $(\mathrm{S} 3) . c e \in(l(S e))(S e)=0$ and $c \in S(1-e)$. Therefore $S(1-e)$ contains a non-zero central idempotent $c$, as desired.

Lemma 4. Let $S$ be a semisimple left injective ring and $A$ a principal left ideal. Then, for any given positive integer $n A$ has a decomposition $A=B \oplus C$ such that (i) $B$ is a direct sum of $n$ mutually isomorphic principal left ideals and (ii) $C$ is a left ideal with $m(C)<n$.

Proof. By virtue of Zorn's lemma it is not too hard to see that there exists a maximal left ideal $B$ of $S$ such that (i) $B \subset A$ and (ii) $B$ is a direct sum of $\mathrm{n}$ mutually isomorphic left ideals $B_{i}$ of $S$. By Lemma 2 there is a minimal injective extension $\hat{B}$ of $B$ such that $\hat{B} \in \mathscr{L}(S)$ and $\hat{B} \subset A$. By (Q2) $\hat{B}=\hat{B}_{1}$ $\oplus \ldots \oplus \hat{B}_{n}$ and $\hat{B}_{i} \simeq \hat{B}_{j}$ by $(Q 1)$. In view of the maximality of $B$ we have $B=\hat{B}$ and $B \in \mathscr{L}(S)$. Let $A=B \oplus C$. If $\mathrm{m}(C) \geqslant \mathrm{n}$, there is a left ideal $B^{\prime}$ 
such that (i) $B^{\prime} \subset C$, (ii) $B^{\prime}=B_{1}{ }^{\prime} \oplus \ldots \oplus B_{n}{ }^{\prime}$ for some left ideals $B_{i}{ }^{\prime}$ and (iii) $B_{i}{ }^{\prime} \simeq B_{j}{ }^{\prime}$ for every i, j. Then, $B \oplus B^{\prime}=\left(B_{1} \oplus B_{1}{ }^{\prime}\right) \oplus \ldots \oplus\left(B_{n}+B_{n}{ }^{\prime}\right)$ $\subset A$ and $B_{i} \oplus B_{i}{ }^{\prime} \simeq B_{j} \oplus B_{j}{ }^{\prime}$. This contradicts the maximality of $B$. Therefore $\mathrm{m}(C)<\mathrm{n}$, as desired.

Lemma 5. Let $S$ be a ring and $\theta$ an endomorphism of the left $S$-module $S$. Suppose that there are mutually isomorphic left ideals $A_{1}, A_{2}$ such that $S=A_{1}$ $\oplus \operatorname{ker} \theta$ and $A_{2}$ is a direct summand of ker $\theta$. Then $\theta$ can be represented as a sum of products of idempotent endomorphisms of the left $S$-module $S$.

Proof. Let $\operatorname{ker} \theta=A_{2} \oplus A_{3}$. Denote by $\epsilon_{1}$ and $\epsilon_{2}$ the projections of $S=A_{1} \oplus \operatorname{ker} \theta$ on $A_{1}$ and ker $\theta$ respectively, and by $\omega$ the given isomorphism of $A_{1}$ onto $A_{2}$. Then we have the following decompositions of $S$ :

$$
\begin{aligned}
& S=A_{1}{ }^{\prime} \oplus A_{2} \oplus A_{3} \text { where } A_{1}{ }^{\prime}=\left\{x+\omega(x) ; x \in A_{1}\right\} ; \\
& S=A_{1} \oplus A_{2}{ }^{\prime} \oplus A_{3} \text { where } A_{2}{ }^{\prime}=\left\{\epsilon_{1} \theta \omega^{-1}(y)+y ; y \in A_{2}\right\} ; \\
& S=A_{1}{ }^{\prime \prime}+\operatorname{ker} \theta \quad \text { where } A_{1}^{\prime \prime}=\left\{x-\epsilon_{2} \theta(x) ; x \in A_{1}\right\} .
\end{aligned}
$$

We shall use the following notations:

$\epsilon_{3}=$ the projection of $S$ on $A_{2}$ with respect to (1);

$\epsilon_{4}=$ the projection of $S$ on $A_{1}$ with respect to (2);

$\epsilon_{5}=$ the projection of $S$ on $\operatorname{ker} \theta$ with respect to $(3)$.

Let $x \in A_{1}$. Since $x=(x+\omega(x))-\omega(x), \epsilon_{3}(x)=-\omega(x)$. From $x=\left(x-\epsilon_{2} \theta(x)\right)+\epsilon_{2} \theta(x)$ we have $\epsilon_{5}(x)=\epsilon_{2} \theta(x)$. Next, let $y \in A_{2}$. Then, since $y=-\epsilon_{1} \theta \omega^{-1}(y)+\left(\epsilon_{1} \theta \omega^{-1}(y)+y\right)$, we see that $\epsilon_{4}(y)=-\epsilon_{1} \theta \omega^{-1}(y)$. Thus, for any $x \in A_{1}, \epsilon_{4} \epsilon_{3} \epsilon_{1}(x)=\epsilon_{4} \epsilon_{3}(x)=\epsilon_{4}(-\omega(x))=-\epsilon_{1} \theta \omega^{-1}(-\omega(x))=$ $\epsilon_{1} \theta(x)$ and $\epsilon_{5} \epsilon_{1}(x)=\epsilon_{5}(x)=\epsilon_{2} \theta(x)$, whence $\left(\epsilon_{4} \epsilon_{3} \epsilon_{1}+\epsilon_{5} \epsilon_{1}\right)(x)=\left(\epsilon_{1}+\epsilon_{2}\right) \theta(x)$ $=\theta(x)$. Evidently $\left(\epsilon_{4} \epsilon_{3} \epsilon_{1}+\epsilon_{5} \epsilon_{1}\right)(\operatorname{ker} \theta)=0=\theta(\operatorname{ker} \theta)$. Therefore we obtain $\theta=\epsilon_{4} \epsilon_{3} \epsilon_{1}+\epsilon_{5} \epsilon_{1}$, as desired.

Lemma 6. Under the assumption of Lemma 5 if $\theta$ itself is an idempotent endomorphism of the left $S$-module $S$, then $\theta$ is a sum of two non-singular endomorphisms of the module.

Proof. Assume that $A_{3}$ and $\omega$ have still the same meaning as in the proof of Lemma 5 , and also that $A_{1}=\theta(S)$ without loss in generality. We consider the following mappings:

$$
\begin{array}{llr}
\sigma(x+y+z)=\left(x+\omega^{-1}(y)\right) & -\omega(x) & +z, \\
\sigma^{\prime}(x+y+z)=-\omega^{-1}(y) & +(\omega(x)+y)+z, \\
\rho(x+y+z)=-\omega^{-1}(y) & +\omega(x) & -z, \\
\rho^{\prime}(x+y+z)=\omega^{-1}(y) & -\omega(x) & -z
\end{array}
$$


for $x \in A_{1}, y \in A_{2}$ and $z \in A_{3}$. Evidently these mappings are endomorphisms of the module $S$. It is easy to verify that $\sigma \sigma^{\prime}=\sigma^{\prime} \sigma=1$ and $\rho \rho^{\prime}=\rho^{\prime} \rho=1$. Since $(\sigma+\rho)(x+y+z)=x=\theta(x+y+z)$, we have $\theta=\sigma+\rho$, as desired.

Lemma 7. Under the assumption of Lemma $5, \theta$ can be represented as a sum of non-singular endomorphisms of the left $S$-module $S$.

Proof. From Lemma 5 it follows that $\theta=\epsilon_{4} \epsilon_{3} \epsilon_{1}+\epsilon_{5} \epsilon_{1}$. Now we note that each of the idempotents $\epsilon_{1}, \epsilon_{3}, \epsilon_{4}$, and $1-\epsilon_{5}$ satisfies the assumption for $\theta$ in Lemma 5. Therefore these idempotents are represented as sums of nonsingular endomorphisms. This implies that $\theta$ also can be represented as a sum of non-singular endomorphisms, completing the proof.

THEOREM 2. Let $S$ be a semisimple left self-injective ring with no ideals of index 1. Then $S$ is generated by idempotents, and is also generated by nonsingular elements.

Proof. Let $x \in S$. Then $l(x) \in \mathscr{L}(S)$ by Lemma 1 , and hence $l(x)=S e_{1}$ and $S=S e_{1} \oplus S e_{1}{ }^{\prime}$ for some $e_{1}, e_{1}{ }^{\prime} \in S$. Applying Lemma 4 we obtain a decomposition $S e_{1}{ }^{\prime}=S e_{2} \oplus S e_{3} \oplus S e_{4}$ such that $S e_{2} \simeq S e_{3}$ and $\mathrm{m}\left(S e_{4}\right)<2$. Thus, $S=S e_{1} \oplus S e_{2} \oplus S e_{3} \oplus S e_{4}$. With no loss of generality we assume that $e_{1}, e_{2}, e_{3}$, and $e_{4}$ are orthogonal idempotents and $1=e_{1}+e_{2}+e_{3}+e_{4}$. Evidently, $l\left(e_{2} x\right) \supset S e_{1} \oplus S e_{3} \oplus S e_{4}$. However, if $y \in l\left(e_{2} x\right)$, then $y e_{2} x=0$ and $y e_{2} \in l(x)=S e_{1}$, hence $y e_{2}=y e_{2} e_{1}=0$, whence

$$
y=y\left(e_{1}+e_{2}+e_{3}+e_{4}\right)=y e_{1}+y e_{3}+y e_{4} \in S e_{1}+S e_{3}+S e_{4} .
$$

Thus,

$$
l\left(e_{2} x\right)=S e_{1} \oplus S e_{3} \oplus S e_{4}
$$

Similarly,

$$
l\left(e_{3} x\right)=S e_{1} \oplus S e_{2} \oplus S e_{4},
$$

and

$$
l\left(e_{4} x\right)=S e_{1} \oplus S e_{2} \oplus S e_{3} .
$$

Denote by $T$ the subring of $S$ which is generated by all idempotents (nonsingular elements) of $S$. Since $l\left(e_{2} x\right)$ contains a direct summand $S e_{3}$ isomorphic to $S e_{2}$, Lemma 5 (Lemma 7 ) assures that $e_{2} x \in T$. Also, we have $e_{3} x \in T$ in an analogous way. Next, we shall show that

$$
S e_{1}+S e_{2}+S e_{3}\left(=S\left(e_{1}+e_{2}+e_{3}\right)\right)
$$

contains a left ideal isomorphic to $S e_{4}$. In fact, if not, $e_{4} \neq 0$ and $\mathrm{m}\left(S e_{4}\right)=1$. Then, by virtue of Lemma $3, S\left(1-\left(e_{1}+e_{2}+e_{3}\right)\right)\left(=S e_{4}\right)$ contains a nonzero central idempotent $c$. Of course, $m(S c)=1$ since $0 \neq S c \subset S e_{4}$. Hence by (S1) $S c$ is an ideal of index 1 , which contradicts our assumption. This 
implies that $S e_{1}+S e_{2}+S e_{3}\left(=l\left(e_{4} x\right)\right)$ contains a left ideal $A$ isomorphic to $\mathrm{Se}_{4}$. Since $A \in \mathscr{L}(S)$ it follows from Lemma 5 (Lemma 7 ) that $e_{4} x \in T$. Therefore,

$$
x=x e_{1}+x e_{2}+x e_{3}+x e_{4} \in T
$$

and $S=T$, which completes the proof.

The following examples will illustrate that Theorem 2 does not hold for semisimple self-injective rings of index 1 .

Example 1. Every division ring $D$ is, of course, semisimple self-injective. However, if $D \neq \mathrm{GF}(\mathrm{p})$ for every prime $\mathrm{p}, D$ is not generated by idempotents.

Example 2. Let $M$ be a set containing at least two elements. Then it is well known that the set of all subsets of $M$ forms a complete Boolean algebra $S$. By virtue of (2, Corollary 4$)$ the Boolean ring $S$ is self-injective. However, if $x$ is non-singular, then $x=x\left(x x^{-1}\right)=x^{2} x^{-1}=x x^{-1}=1$. Since $1+1=0$, the subring $T$ generated by all non-singular elements consists of 1 and 0 . Thus, $S \neq T$.

\section{Injectivity of continuous regular rings.}

LEMma 8. Let $S$ be a left continuous regular ring, and $\bar{S}$ the maximal left quotient ring of $S$. Then every idempotent of $\bar{S}$ is contained in $S$.

Proof. Let $e$ be an idempotent of $\bar{S}$, and let $A$ be the set of all idempotents in $\bar{S} e \cap S$. By (Q3), $\bar{S}$ is a regular ring with unit 1 and $\mathscr{L}(\bar{S})$ is complete. Hence there exists the join $\bigcup_{f \in A} \bar{S} f$. Clearly $\bar{S} e \supset \bigcup_{f \in A} \bar{S} f$, and $\bar{S} e=\left(\cup_{f \in A} \bar{S} f\right) \oplus$ $\bar{S} g$ for some $g \in \bar{S}$. If $\bar{S} g \neq 0$, then $\bar{S} g \cap S \neq 0$ by (Q4), whence $\bar{S} g \cap S$ contains an idempotent $f^{\prime} \neq 0$. Since $f^{\prime} \in \bar{S} g \cap S \subset \bar{S} e \cap S$, we have $f^{\prime} \in A$, and so $f^{\prime} \in\left(\cup_{f \in A} \bar{S} f\right) \cap \bar{S} g=0$, a contradiction. Thus, $\bar{S} g=0$ and $\bar{S} e=$ $\cup_{f \in A} \bar{S} f$. On the other hand, by Lemma 2 there is an idempotent $e^{\prime} \in S$ such that $\sum_{f \epsilon A} S f \subset^{\prime} S e^{\prime}$. Evidently $\bar{S} e^{\prime} \supset \cup_{f \epsilon A} \bar{S} f=\bar{S} e$. Let $\bar{S} e^{\prime}=\bar{S} e \oplus \bar{S} h$. Then,

$$
S e^{\prime}=\bar{S} e^{\prime} \cap S \supset(\bar{S} e \cap S) \oplus(\bar{S} h \cap S) \supset\left(\sum_{f \in A} S f\right) \oplus(\bar{S} h \cap S) .
$$

Since $\sum_{f \epsilon A} S f \subset^{\prime} S e^{\prime}$, it follows that $\bar{S} h \cap S=0$. Hence $\bar{S} h=0$ by (Q4), and we see that $\bar{S} e=\bar{S} e^{\prime}$. This shows that every principal left ideal of $\bar{S}$ is generated by an idempotent in $S$. In particular, $\bar{S}(1-e)=\bar{S} e^{\prime \prime}$ for some idempotent $e^{\prime \prime} \in S$. Let $S e^{\prime} \oplus S e^{\prime \prime}=S u, u$ being an idempotent. By (Q5) the unit 1 of $\bar{S}$ is contained in $S$, and so $\bar{S} S=\bar{S}$. Hence we have

$$
\bar{S} u=\bar{S} e^{\prime} \oplus \bar{S} e^{\prime \prime}=\bar{S} e \oplus \bar{S}(1-e)=\bar{S} .
$$

This implies by (S3) that $u$ is central and hence that $u=1$. Thus, $S e^{\prime} \oplus S e^{\prime \prime}=$ $S$. Let $x+y=1, x \in S e^{\prime}, y \in S e^{\prime \prime}$. Since $x \in S e^{\prime} \subset \bar{S} e^{\prime}=\bar{S} e$ and $y \in S e^{\prime \prime} \subset$ $\bar{S} e^{\prime \prime}=\bar{S}(1-e)$, we know that $x=x e$ and $y e=0$. Hence, $e=(x+y) e=$ $x e=x \in S e^{\prime} \subset S$. Therefore every idempotent $e$ of $\bar{S}$ belongs to $S$, as desired. 
TheOREm 3. Let $S$ be a left continuous regular ring with no ideals of index 1 . Then $S$ is left self-injective.

Proof. Denote the maximal left quotient ring of $S$ by $\bar{S}$. If $\bar{S}$ has an ideal $A$ of index 1 , then $A \cap S \neq 0$ by (Q4). Hence $\mathrm{i}(A \cap S)=1$ and we have a contradiction to the assumption. Thus, $\bar{S}$ has no ideals of index 1 . Since $\bar{S}$ is semisimple left self-injective by (Q3) it follows from Theorem 2 that $\bar{S}$ is generated by idempotents. Now, Lemma 8 assures that every idempotent of $\bar{S}$ is contained in $S$. Therefore, $\bar{S}=S$ and $S$ also is left self-injective.

COROllary. Every continuous regular ring with no ideals of index 1 is self-injective.

The following example will illustrate the existence of continuous regular rings which are not left (right) self-injective.

Example 3. Let $\left\{D_{\alpha}\right\}$ be an infinite family of division rings $D_{\alpha}$, and let $F_{\alpha}$ be a proper division subring of $D_{\alpha}$ for each $\alpha$. We denote by $S$ the subring of the complete direct sum of $D_{\alpha}$ consisting of all elements $x$ such that all but a finite number of $\alpha$-components of $x$ belong to $F_{\alpha}$. Then $S$ is a continuous regular ring. However, the minimal left self-injective (right self-injective, self-injective) extension ring of $S$ is the complete direct sum of $D_{\alpha}$.

5. Ideals of index 1. In connection with the assumption of Theorems 2 and 3 it may be of interest to see that every left continuous regular ring $S$ has the decomposition $S=S_{1} \oplus S_{1}{ }^{\prime}$ such that $S_{1}$ is, if non-zero, an ideal of index 1 and $S_{1}{ }^{\prime}$ is an ideal not containing any ideals of index 1 . This is an immediate consequence of the following

Theorem 4. Let $S$ be a semisimple I-ring. Then $S$ has a maximal ideal $S_{n}$ of index $\leqslant n . l\left(\mathrm{r}\left(S_{n}\right)\right)=S_{n}$.

Proof. Let $S_{n}$ be the sum of all ideals of index $\leqslant \mathrm{n}$, and let $a$ be a nilpotent element of $l\left(\mathrm{r}\left(S_{n}\right)\right)$. If the index of $a$ is $\mathrm{m}, l\left(\mathrm{r}\left(S_{n}\right)\right)$ contains a system $\left\{e_{i j}\right\}$ of total matrix units of degree $m$ by virtue of (6, Theorem 1, p. 237). Assume that $S e_{11} \cap A=0$ for every ideal $A$ of index $\leqslant \mathrm{n}$. Then $A S e_{11} \subset S e_{11} \cap A=0$, and $A S e_{11}=0$, whence $S_{n} S e_{11}=0$ and $S e_{11} \subset \mathrm{r}\left(S_{n}\right)$. Thus, $\left(S e_{11}\right)^{2} \subset l\left(\mathrm{r}\left(S_{n}\right)\right) \mathrm{r}$ $\left(S_{n}\right)=0$ and so $e_{11}=0$, a contradiction. Therefore, $S e_{11} \cap A \neq 0$ for some ideal $A$ of index $\leqslant$ n. By assumption $S e_{11} \cap A$ contains a nonzero idempotent $f_{11}{ }^{\prime}$. Set $f_{i j}=e_{i 1} f_{11}{ }^{\prime} e_{1 j}$. Then it is easy to verify that $\left\{f_{i j}\right\}$ forms a system of total matrix units of degree $\mathrm{m}$. Hence $\sum f_{i i+1}$ is a nilpotent element of index m. Since $f_{11}{ }^{\prime} \in A$, we have $f_{i j} \in A$ and $\sum f_{i i+1} \in A$. Thus, $\mathrm{m} \leqslant \mathrm{n}$ and so $\mathrm{i}\left(l\left(\mathrm{r}\left(S_{n}\right)\right) \leqslant \mathrm{n}\right.$. Therefore, $l\left(\mathrm{r}\left(S_{n}\right)\right)=S_{n}$ and $\mathrm{i}\left(S_{n}\right) \leqslant \mathrm{n}$.

COROLlary. Let $S$ be a regular ring and suppose that $\mathscr{L}(S)$ is complete. Then, for every positive integer $n$ there is the decomposition $S=S_{n} \oplus S_{n}{ }^{\prime}$ such that $S_{n}$ is an ideal of index $\leqslant n$ and $S_{n}{ }^{\prime} i$ s an ideal not containing any ideals of index $\leqslant n$. 
Proof. By Theorem 4 the maximal ideal $S_{n}$ of index $\leqslant \mathrm{n}$ is a left annihilator ideal. Hence, by Lemma $1, S_{n}$ is a principal left ideal. Moreover, since $S_{n}$ is an ideal, (S3) assures that $S_{n}$ is generated by a central idempotent. Thus, $S=S_{n} \oplus S_{n}{ }^{\prime}$ for some ideal $S_{n}{ }^{\prime}$. If $S_{n}{ }^{\prime}$ contains an ideal $A$ of index $\leqslant \mathrm{n}$, then $A$ is also an ideal of $S$ and hence is contained in $S_{n}$. This implies that $A \subset S_{n} \cap$ $S_{n}{ }^{\prime}=0$, completing the proof.

Theorem 5. Let $S$ be a regular ring with unit, and let $\mathrm{i}(S)=1$. Then the following properties are equivalent:

(i) $S$ is continuous.

(ii) The Boolean algebra $B$ of idempotents of $S$ is complete.

(iii) The Boolean ring $B$ of idempotents of $S$ is self-injective.

Proof. Every idempotent of $S$ is central by (S2). Hence the set $B$ of all idempotents of $S$ forms a Boolean algebra by (R3). Moreover, every principal (left) ideal $A$ is generated by a central idempotent $e_{A}$. Thus, it is easy to see that the correspondence $A \rightarrow e_{A}$ gives an isomorphism of $\mathscr{L}(S)(=\mathscr{R}(S))$ and $B$. If $S$ is continuous, then $\mathscr{L}(S)$ is complete and so is $B$. Conversely, if $B$ is a complete Boolean algebra, $B$ satisfies the infinite distributivity (1, p. 165), and hence $B$ is upper continuous. Therefore, $\mathscr{L}(S)(=\mathscr{R}(S))$ is upper continuous, and $S$ is continuous.

(ii) $\rightarrow$ (iii) follows directly from (2, Corollary 4 ). If we assume (iii), then $B$ is the maximal quotient ring of $B$ itself by $(Q 4)$. Hence $B$ is complete by (2, Theorem 5), as desired.

\section{REFERENCES}

1. G. Birkhoff, Lattice theory, Amer. Math. Soc. Colloq. Publ., 25, rev. ed. (1948).

2. B. Brainerd and J. Lambek, On the ring of quotients of a Boolean ring, Can. Math. Bull., 2 (1959), 25-29.

3. H. Cartan and S. Eilenberg, Homological algebra (Princeton, 1956).

4. B. Eckmann and A. Schopf, Ueber injektive Moduln, Archiv der Mathematik, 4 (1956), 75-78.

5. G. D. Findlay and J. Lambek, A generalized ring of quotients, Can. Math. Bull., 1 (1958), $77-85,155-167$.

6. N. Jacobson, Structure of rings, Amer. Math. Soc. Colloq. Publ., 37 (1956).

7. R. E. Johnson, The extended centralizer of a ring over a module, Proc. Amer. Math. Soc., 2 (1951), 891-895.

8. F. Maeda, Kontinuierliche Geometrien (Springer, 1958).

9. J. von Neumann, Continuous geometry, Part I (Princeton, 1936).

10. Y. Utumi, On quotient rings, Osaka Math. J., 8 (1956), 1-16.

11. - A note on an inequality of Levitzki, Proc. Japan Acad., 33 (1957), 249-251.

12. - On a theorem on modular lattices, Proc. Japan Acad., 35 (1959), 16-21.

13. K. G. Wolfson, An ideal theoretic characterization of the ring of all linear transformations, Amer. J. Math., 75 (1957), 358-386.

14. E. T. Wong, On injective rings, Bull. Amer. Math. Soc., 63 (1957), 104.

15. D. Zelinsky, Every linear transformation is a sum of non-singular ones, Proc. Amer. Math. Soc., 5 (1954), 627-630.

O saka Women's University and McGill University 